\title{
CircRNAs: From anonymity to novel regulators of gene expression in cancer (Review)
}

\author{
KATHERINE L. HARPER ${ }^{1}$, EUAN MCDONNELL ${ }^{1}$ and ADRIAN WHITEHOUSE ${ }^{1,2}$ \\ ${ }^{1}$ School of Molecular and Cellular Biology; ${ }^{2}$ Astbury Centre for Structural Molecular Biology, \\ Faculty of Biological Sciences, University of Leeds, Leeds, LS2 9JT, UK
}

Received May 23, 2019; Accepted July 31, 2019

DOI: $10.3892 /$ ijo.2019.4904

\begin{abstract}
Circular RNAs (circRNAs) are a group of non-coding RNAs, formed mostly through a unique backsplicing mechanism. Originally proposed to be a by-product from errors in splicing, recent studies have shown they exhibit a range of roles in regulating gene expression, including sponging of microRNAs (miRNAs), interactions with RNA-binding proteins and regulation of transcription. Though research is still in its infancy, evidence suggests circRNA levels are tightly regulated in the cell, reinforced by dysregulated circRNAs levels being implicated in a range of diseases, including cancer and viral infection. There is growing interest in circRNAs playing specific roles in cancers, either oncogenic or as tumour suppressors, with particular focus on their potential as novel biomarkers. This review will provide an overview of circRNA biogenesis and regulation, and their potential roles in the cell, with a focus on their dysregulation in cancer.
\end{abstract}

\section{Contents}

1. Introduction

2. Biogenesis

3. Regulation and dysregulation of circRNAs

4. Functions

5. miRNA sponges

6. Transcriptional regulators

7. Interactions with proteins

8. Translated circRNAs

9. Oncogenic Viruses and circRNAs

10. circRNAs and exosomes

11. Biomarkers

12. Fusion circRNAs (f-circRNAs)

13. Future Prospects

Correspondence to: Professor Adrian Whitehouse, School of Molecular and Cellular Biology, Faculty of Biological Sciences, University of Leeds, Leeds, LS2 9JT, UK

E-mail: a.whitehouse@leeds.ac.uk

Key words: circular RNAs, biogenesis, function, cancer

\section{Introduction}

Circular RNAs (circRNAs) are a group of non-coding RNAs that in recent years have emerged as potential regulators of various cell processes. It is proposed that they serve a range of functions, including regulation of RNA-binding proteins (RBPs), promotion of mRNA cis-transcription and post-transcriptional regulation through sponging of microRNAs (miRNAs/miRs) (1). They may also interact with splicing proteins or RNA polymerase II (RNA pol II) to aid in transcriptional regulation (2). Due to their numerous and varied regulatory roles, circRNAs have been postulated to serve key roles in cancer, acting in both oncogenic and tumour-suppressive manners. Although research focusing on circRNAs in cancer remains in its infancy, circRNAs have already been discovered to be dysregulated in a number of different cancers, as well as other types of diseases.

RNA was first discovered to be able to form natural circles $>40$ years ago in both plant viroids and in hepatitis $\delta$ virus (3). In 1991, circRNAs were found in human cells when circular isoforms with out-of-order exons from the gene $D C C$ (encoding 'deleted in colorectal cancer' protein) were discovered, however, they were dismissed as a by-product from errors in RNA processing and splicing (4). Throughout the 1990s and early 2000s, increasing evidence for the existence of circRNAs in mammalian cells, such as transcripts with out-of-order exons, were discovered, although they were still considered to be low-abundance and non-functional RNAs (5). Improvements in RNA sequencing techniques have since revealed that circRNAs are expressed at far higher levels than initially considered, whereas the discovery in 2013 of a circRNA that was capable of sponging miRNAs led to the increased interest in their roles (6). This key finding provided evidence that circRNAs serve a functional role as master regulators of gene expression and are not simple by-products of splicing (7).

\section{Biogenesis}

The biogenesis of circRNAs is yet to be fully elucidated; however, most are formed through backsplicing. Unlike canonical splicing, backsplicing results in the 3 ' splice donor site being joined to the upstream 5 ' splice acceptor site forming a closed circle (8). These circRNAs are characterised as either 
exonic circRNAs (Ecircs), exonic-intronic circRNAs (EIcircs) or circular intronic circRNAs (CIcircs) (9). The vast majority of circRNAs discovered are Ecircs, with 2 main models for formation: Intron-pairing or lariat-driven (Fig. 1) (10).

In intron-pairing, circularisation is driven by flanking inverted repeats in the introns either side of the exons. These repeats, particularly ALU elements, promote circRNA formation through base pairing of the introns bringing backsplicing sites closer together, enabling trans factors to facilitate circularisation (11). Lariat-driven formation occurs through exon skipping; this is a form of alternative splicing where exons are spliced from the main transcript, allowing greater diversity (12). These skipped exons can form a lariat with the removed introns; under certain conditions, before this lariat is degraded, it can undergo further splicing to remove the introns, forming a circRNA from the spliced exons (13). Similarly to intron-pairing, formation of a lariat drives circularisation through bringing the backsplice sites closer together, although the specific cis and trans factors that aid circularisation are not known.

Similar to exon-containing circRNAs, intron only-containing circRNA formation is promoted through $\mathrm{cis}$ factors bringing splice sites into close proximity (14). With intron-containing circRNAs, these cis factors contain two consensus motifs. These motifs are a 7-nucleotide GU-rich element at the 5' end and an 11-nucleotide C-rich element at the branch site; together, these motifs bind and promote the formation of an intermediate circular lariat. These lariats escape degradation before further processing removes any exons or overhangs on the lariat, leading to the formation of a stable intron-only circRNA (15).

\section{Regulation and dysregulation of circRNAs}

The regulation of circRNAs during and after transcription remains to be fully elucidated, with various complex factors controlling their transcription and splicing. Specific RBPs can promote circRNA formation; for example, the mannose-binding lectin ( $\mathrm{Mbl}$ ) protein can bind to the flanking introns of circRNAs, acting as a bridge to bring the splice sites closer and promote circularisation (16). Another RBP termed Quaking (QKI) has also been demonstrated to regulate circRNA formation (17). QKI is hypothesised to specifically regulate multiple circRNAs involved in the epithelial-mesenchymal transition (EMT). QKI regulation, similar to Mbl, requires binding motifs within the flanking introns (17). These motifs are sufficient to promote circularisation, with the addition of these motifs to linear transcripts leading to circRNA formation. QKI protein binds to these motifs, forming a dimer structure, bringing the splice sites closer together to promote circularisation (17).

The nuclear export of Ecircs is also regulated, although the mechanism is yet to be fully determined. A recent study has suggested that the nuclear export of circRNAs is size-dependent (18). Using an RNA interference-mediated approach targeting known nuclear export-related proteins, the impact on circRNA localization was evaluated in Drosophila DL1 cells. Results showed that depletion of the DExH/D-Box helicase, helicase at 25E (Hel25E), resulted in nuclear accumulation of circRNAs which were $>800$ nucleotides in length. Of note, it was further demonstrated that homologues of Hel25E, namely UAP56/DDX39B and URH49/DDX39A, have a similar role in mammalian cells. Longer circRNAs are exported through UAP56, whilst shorter circRNAs are exported through URH49 in humans (18). At present, the protein which exports shorter circRNAs in Drosophila remains to be determined. The regulation of circRNA nuclear export by these RNA helicases may provide a novel therapeutic approach to prevent circRNA dysregulation, as small molecule inhibitors are being developed which inhibit UAP56 export ability (19).

Negative regulators of circRNA formation have also been identified; these include the double-strand RNA editing enzyme adenosine deaminase acting on RNA 1 (ADAR1) and the enzyme RNA Helicase A (DHX9). Overexpression of ADAR leads to significantly decreased levels of circRNAs; this mechanism is proposed to occur due to disruption of base pairing of the flanking introns (20). DHX9 is hypothesised to have roles in the regulation of ALU elements by binding and preventing secondary structure formation (21). As ALU secondary structures aid in promoting circularisation by bringing flanking introns in closer proximity, through this binding, DHX9 can also suppress circRNA formation (11). Furthermore, other ALU element regulators, such as heterogeneous nuclear riboprotein ( $h n R N P$ ) $\mathrm{C}$ and hnRNP L, have also been shown to affect circRNA expression (7).

Studies have found circRNAs are frequently dysregulated in cancer, although exact mechanisms are not fully elucidated (22-25). However, one known mechanism is through mutations in flanking introns; certain cancers, such as gastric cancer, often have flanking intron mutations; these can lead to mismatches or shortenings of introns, leading to reduced circRNA biogenesis (26). Dysregulation of proteins that have functions in regulating circRNA biogenesis has also been discovered; for example, ADAR is commonly found dysregulated in cancers, from hepatocellular carcinoma (HCC) and lung cancer to melanoma (27). Through ADAR, base changes in flanking introns could lead to dysregulated biogenesis. Both hnRNPs and DHX9 have also found to be dysregulated in cancer, with both dysregulated in breast and lung cancers $(28,29)$.

There is also the potential for aberrant expression of transcription factors or splicing factors in cancer affecting circRNA levels. Although the role of splicing and transcription in relation to circRNAs is not fully elucidated, key mutations could lead to changes. For instance, the transcription factor $\mathrm{C}$-myc frequently acts an oncoprotein and is upregulated in cancer; it is able to regulate specific circRNAs through myc-binding sites in circRNA promoters (30).

\section{Functions}

The proposed functions of circRNAs in regulating gene expression include miRNA sponging, interaction with RBPs and regulation of gene transcription (Fig. 2). Ecircs are proposed to carry out the majority of miRNA sponging and interaction with RBPs, whereas CIcircs and EIcircs are hypothesised to be involved in transcriptional control. Whether this division of function is due to the composition of the circRNAs or (more likely) their subcellular localisation, is unknown, with Ecircs generally found in the cytoplasm and CI/EIcircs located in the nucleus. 

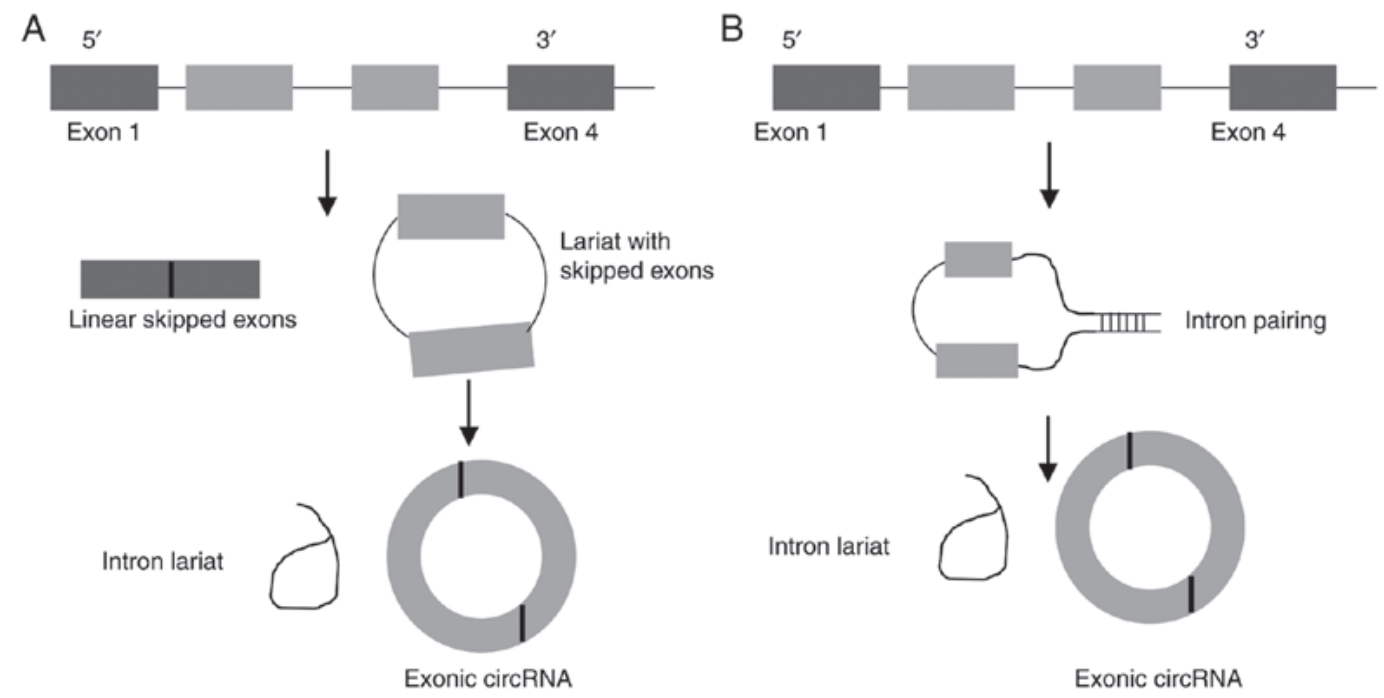

Figure 1. Two main models for the biogenesis of exonic circRNAs. (A) Lariat formation through exon skipping. Exon skipping occurs, forming a linear by-product, and a lariat containing the skipped exons and introns. This lariat then undergoes further splicing to remove the introns from the lariat and form an exonic circRNA. (B) circRNA formation through intron pairing. Complementary sequences between flanking introns bring the upstream 5 ' site and downstream 3 ' site in close proximity, promoting a backsplice event and the formation of an exonic circRNA. circRNA, circular RNA.

\section{5. miRNA sponges}

It is hypothesised that one of the main functions of circRNAs is to act as miRNA sponges, acting as endogenous regulators of miRNA activity (31). miRNAs are involved in post-transcriptional regulation of gene expression through binding to complementary mRNAs and instigating translational inhibition by degradation or P-body storage of the mRNA (32). Through sponging of the miRNAs it is hypothesised that circRNAs play a key role in regulating gene expression and are often found to be dysregulated in diseases associated with aberrant gene expression (33). Through sponging of miRNAs, circRNAs act as crucial post-transcriptional regulators and play a key role in a regulatory network involving circRNAs, miRNAs and mRNAs. As they regulate through competition, circRNAs represent a novel class of competing endogenous RNA (ceRNA) (34). Compared with other known ceRNAs, circRNAs are more abundant and stable, and can contain a number of binding sites for miRNAs, allowing efficient regulation (35). Due to this regulation, circRNAs may play a key regulatory role in diseases associated with dysregulated miRNAs, including cancer, Parkinson's disease, hypertension and viral infection (36-38). This is supported by the fact that numerous circRNAs have already been reported as dysregulated in cancer; however, it must be noted that numerous circRNAs lack miRNA binding sites, with one study finding only $12 \%$ of circRNAs studied contained miRNA binding sites and an argonaute (AGO) footprint (39). Furthermore, a number of the circRNAs that do contain miRNA binding sites have far fewer than the notable examples, such as ciRS-7 (also known as CDR1as) and sex-determining region Y circRNA $(40,41)$.

The first instance of circRNAs sponging miRNAs was reported in 2013, when the circRNA derived from the CDRI gene, ciRS-7, was shown to sponge miR-7 (6). ciRS-7 contains $>70$ binding sites for miR-7, with $90 \%$ of its sequence composed of hexamer repeats that comprise the miR-7 binding site (42). The use of AGO-crosslinking/immunoprecipitation (IP) has been integral for the confirmation of circRNAs as miRNA sponges. AGO is a key protein member of the RNA-induced silencing complex (RISC) involved in miRNA function (43). miRNA-sponging circRNAs precipitate strongly with AGO2. Furthermore, circRNAs are enriched in AGO-IPs upon overexpression of target miRNAs (44). As such, the target miRNA facilitates complex formation between the circRNA and AGO2, enabling circRNA sponging ability and high occupancy of AGO sites (6). ciRS-7 interacts with miR-7, sequestering it from its target mRNA. Originally, ciRS-7 was found to act in the neuronal cells of zebrafish embryos and, by sponging miR-7, led to brain development defects via dysregulation of the mTOR pathway (45). Outside the brain, other miR-7 target mRNAs include epidermal growth factor receptor (EGFR) and X-linked inhibitor of apoptosis protein, two proteins frequently dysregulated in cancer (46). Furthermore, ciRS-7 has been found to be upregulated in pancreatic ductal adenocarcinoma (PDAC), where it aids in promoting proliferation through the sponging of miR-7, leading to increased activity of the EGFR/STAT3 pathway (47). By sponging miR-7, ciRS-7 acts as an oncogenic circRNA, allowing increased levels of several proteins that promote cell proliferation and negatively regulate apoptosis (45).

One of the most extensively studied dysregulated circRNAs is homeodomain interacting protein kinase 3 circRNA (circHIPK3), a 1099-base pair circRNA formed from exon 2 of the HIPK3 gene (48). Through sequence analysis, it has been identified that circHIPK3 may contain 18 binding sites for 12 different miRNAs, including binding sites for miR-7, miR-29b and miR-124 that have been confirmed (48-50). Through the sponging of miR-7, circHIPK3 leads to an increase in the expression of focal adhesion kinase (FAK), EGFR, YY1 transcription factor and insulin-like growth factor 1 receptor (49). These oncogenes can promote metastasis and tumour growth by activating the PI3K/AKT and mitogen-activated protein kinase kinase/ERK pathways, increasing cell proliferation and leading to an increase in expression of pro-metastatic proteins, 


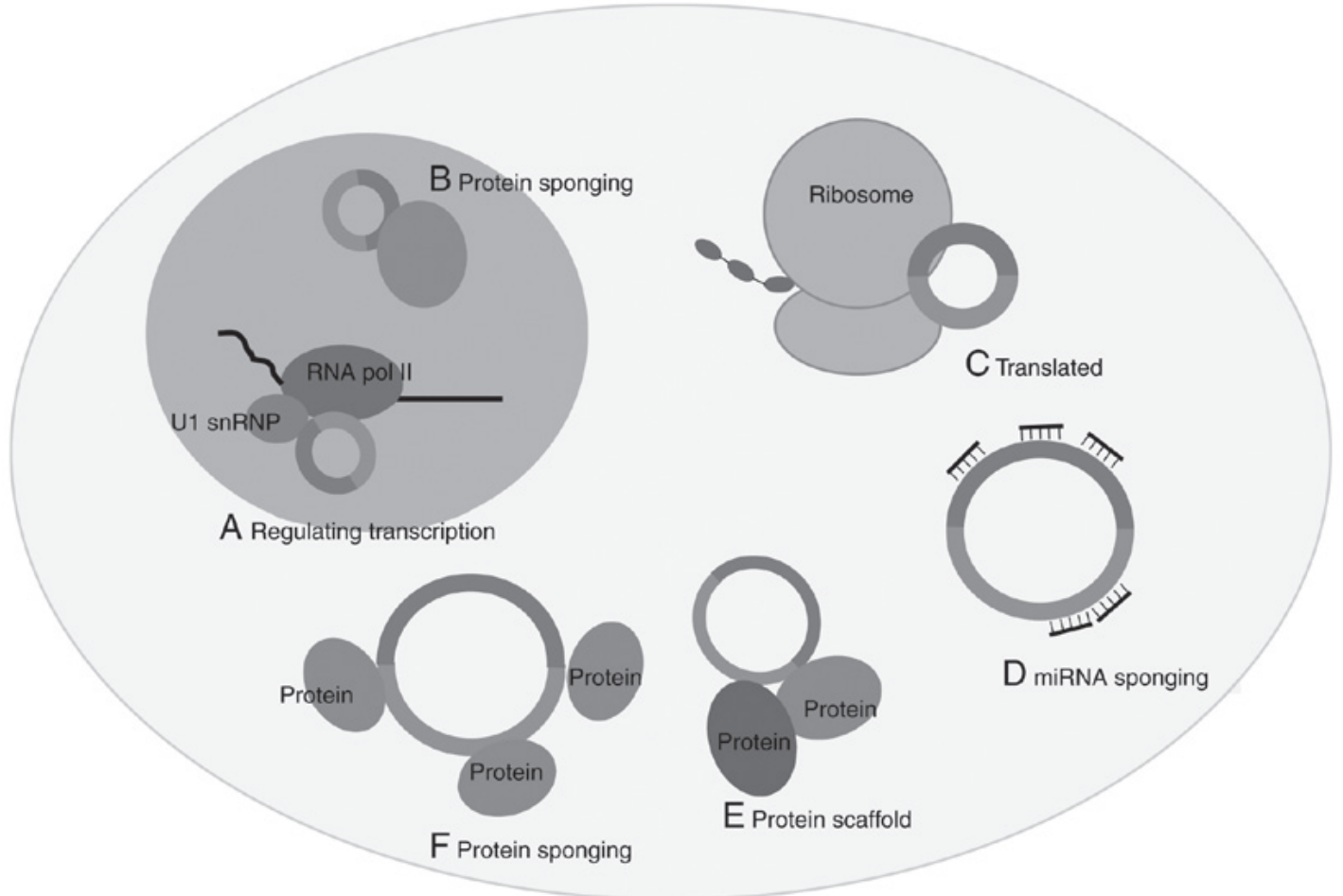

Figure 2. Proposed functions for circRNAs. (A) Regulating gene expression through associations with RNA pol II and U1 snRNP, increasing transcription. (B) Sponging RNA-binding proteins in the nucleus, preventing their normal function. (C) CircRNAs can potentially be translated. (D) Sponging miRNAs to regulate gene expression. (E) Sponging of proteins in the cytoplasm. (F) circRNAs acting as a protein scaffold, facilitating protein-protein interactions. circRNA, circular RNA; miRNA, microRNA; RNA pol II, RNA polymerase II; snRNP, small nuclear ribonucleoprotein.

including vascular endothelial growth factor, and metalloproteinase (MMP)2 and MMP9 (49). circHIPK3 also sponges the tumour suppressor miR-124, leading to an increase in the expression of miR-124 target mRNAs, including STAT3 and cyclin-dependent kinase 4 (CDK4), both commonly found to be upregulated in cancer (51). STAT3 is a transcription factor promoting cell growth through the activation of pro-cell cycle progression proteins and anti-apoptotic proteins, while CDK4 promotes cell cycle progression $(51,52)$. circHIPK3 has now been found to be upregulated in a range of cancers, including $\mathrm{HCC}$, and colorectal, gastric, prostate and lung cancers, and may represent a novel biomarker or therapeutic target $(22,50,53)$. Studies have shown some promise with silencing circHIPK3, reducing the proliferation of cancer cells and inducing apoptosis, whereas overexpression constructs promoted cell survival $(49,51)$.

circRNAs can also act as tumour suppressors via the sponging of oncomiRs, thereby decreasing the expression of oncoproteins. La-related protein 4 circRNA (circLARP4) sponges miR-424, leading to an increase in the expression of serine/threonine-protein kinase LATS1, which in turn downregulates the pro-proliferative yes-associated protein (YAP) pathway (Fig. 3) (54). Decreased expression of circLARP4 was found to be associated with poorer prognosis and survival in gastric cancer (54). A second study also reported that low circLARP4 expression was associated with a poorer prognosis in HCC, with overexpression of circLARP4 leading to an increase in the levels of p53 and p21 (55). The study suggested that circLARP4 sponges miR-761, leading to upregulation of its target runt-related transcription factor 3 (RUNX3), which in turn activates downstream p53 and p21 signalling. RUNX3 is a transcription factor that has previously been reported to function as a tumour suppressor, with loss of expression associated with gastric cancer, although there is a degree of controversy as to whether RUNX3 possesses tumour-suppressive or oncogenic properties (56).

An unusual example of post-transcriptional regulation via circRNA sponging of miRNAs is itchy E3 ubiquitin protein ligase circRNA (circITCH), as circITCH regulates the expression of the linear transcript it is derived from: The ITCH gene (57). circITCH contains binding sites for miR-7, miR-17 and miR-214 (42). These miRNA seed sequences are found in the 3' untranslated region (UTR) of the linear ITCH transcript, suggesting the miRNA may suppress linear ITCH's expression. circITCH sponges these miRNAs, leading to an increase in the mRNA expression of its linear form. ITCH functions as an E3 ubiquitin ligase, targeting proteins for degradation; one such protein is dishevelled segment polarity protein 2 (Dvl2). Targeting Dvl2 leads to a downregulation of the Wnt/ $\beta$-catenin pathway (57). By upregulating the levels of ITCH mRNA levels (and therefore its protein levels) via miRNA sponging, circITCH may exhibit tumour suppressive properties $(57,58)$. Further examples of miRNAs sponged by circRNAs are presented in Table I (24,25,59-65).

\section{Transcriptional regulators}

A lesser-understood role of circRNAs involves acting as transcriptional regulators. Intron-containing circRNAs are retained in the nucleus, and are hypothesised to regulate the 
A
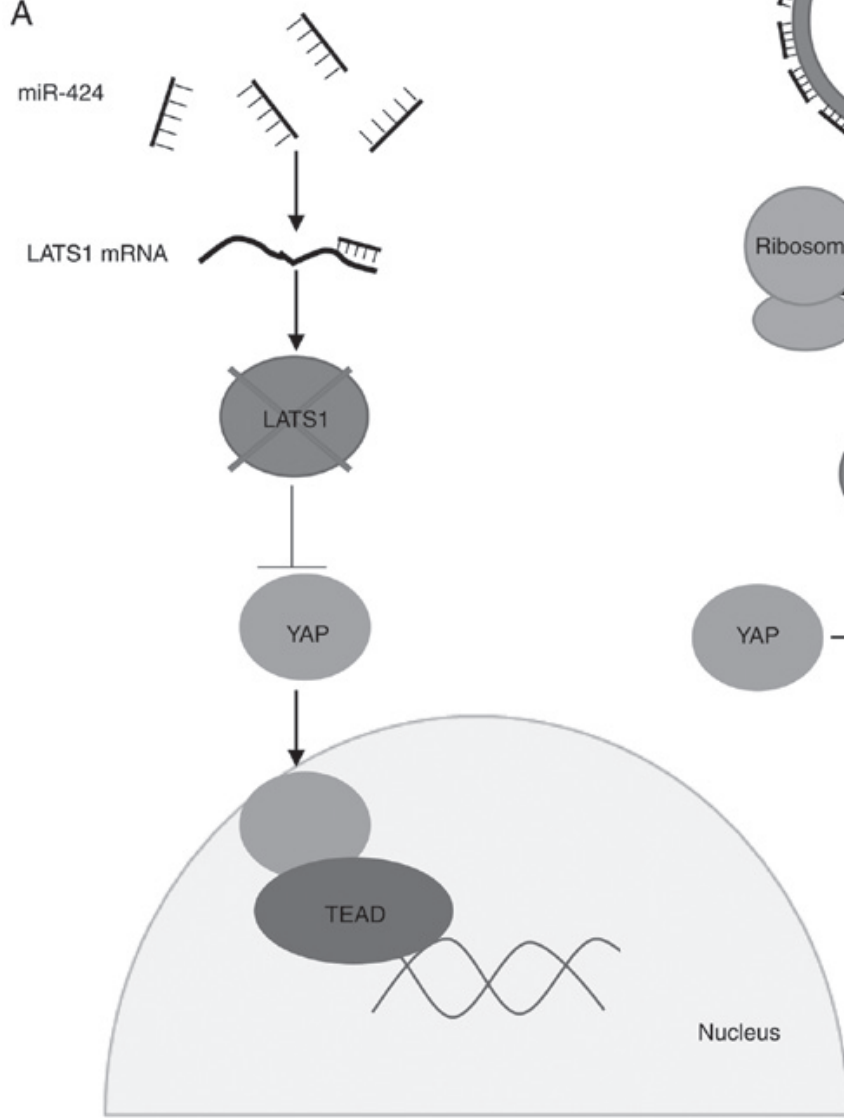

B

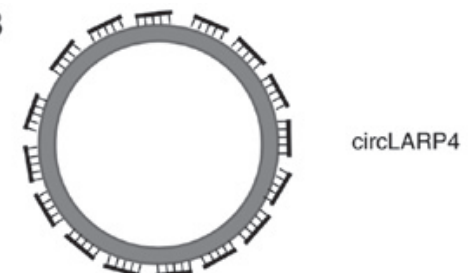

Figure 3. Proposed mechanisms for an miRNA-sponging circRNA, circLARP4. (A) Low levels of circLARP4 lead to increased miR-424, which binds to its target mRNA LATS1, leading to reduced levels. Low levels of LATS1 allow YAP to translocate to the nucleus, where it acts as a transcriptional coactivator through associations with TEAD transcription factors, leading to cell proliferation. (B) Increased circLARP4 sponges miR-424, which leads to increased translation of LATS1. LATS1 phosphorylates YAP, preventing its translocation to the nucleus and inhibiting its activity. circRNA, circular RNA; LARP4, La-related protein 4; miRNA/miR, microRNA; TEAD1, TEA domain family member 1; YAP, yes-associated protein.

transcription of genes. It is proposed that the circRNAs upregulate their parental gene expression through the formation of a circRNA-U1 small nuclear (sn)ribonucleoprotein complex that interacts with RNA pol II (66). Nuclear circRNAs can also interact with additional transcriptional and splicing regulators, including $\mathrm{U} 1 \mathrm{~A}$ and $\mathrm{U} 1 \mathrm{C}$. It has been shown that these circRNAs localise to the loci of their parental genes and co-immunoprecipitate with their promoters, suggesting that they may regulate their expression in cis (9). Conversely, they can also localise to other areas, suggesting some trans regulatory mechanisms $(15,67)$. One circRNA proposed to regulate transcription and splicing within the nucleus is E3 ubiquitin-protein ligase UBR5 circRNA (circUBR5). circUBR5 can bind to QKI, Nova-1 and U1 snRNA, and is therefore hypothesised to serve a regulatory role in splicing, although exact mechanisms remain to be elucidated (68). Notably, circUBR5 has been reported as downregulated in non-small cell lung cancer.

\section{Interactions with proteins}

circRNAs also interact with proteins, acting as scaffolds or sponges (also termed decoys); through this mechanism, they can regulate crucial cellular processes, including ribosome biogenesis and cell cycle progression $(69,70)$. Several of these protein-interacting circRNAs have also been implicated in tumourigenesis. One such circRNA is poly(A) binding protein nuclear 1 circRNA (circPABPN1); this can form complexes with the RBP human antigen $\mathrm{R}$ (HuR), thereby competitively preventing HuR from binding to PABPN1 mRNA (69). This suggests competition between the linear and circular form of PABPN1 serves a role in regulating their expression. PABN1 is required for efficient 3 ' poly(A) tail formation, and therefore mRNA nuclear export and mRNA stability (71). circPABN1, through binding and sponging of the HuR protein, reduces $3^{\prime}$ poly(A) tail formation, leading to a reduction in mRNA export and stability.

Other circRNAs also interact with HuR, including circAGO2; this binding enhances export of HuR to the cytoplasm, where it binds to 3' UTRs of mRNA, leading to reduced AGO2/RISC complex binding and miRNA-mediated translational repression (23). Through this mechanism, circAGO2 promotes cancer metastasis and promotes tumourigenesis both in vitro and in vivo. It has been found upregulated in neuroblastoma, and gastric, colon and prostate cancers, and is associated with a poor prognosis (23). 
Table I. Examples of known circRNA:miRNA:mRNA regulatory networks.

\begin{tabular}{|c|c|c|c|c|}
\hline circRNA & miRNA sponged & miRNA targets & Function regulated & Associated cancer \\
\hline circAKT3 & miR-198 & PIK3R1 & Apoptosis, DNA repair & Gastric (59) \\
\hline circSRY & $\operatorname{miR}-138$ & $\begin{array}{l}\text { RhoC, TWIST2, H2AX, } \\
\text { Bcl-2, SOX } 4\end{array}$ & Migration and invasion & $\begin{array}{l}\text { CRC, } \\
\text { cholangiocarcinoma (60) }\end{array}$ \\
\hline circPVT1 & $\begin{array}{l}\operatorname{miR}-125 a, \operatorname{miR}-125 b \text {, } \\
\text { let-7 }\end{array}$ & HMGA2, KRAS, E2F2 & Cell proliferation, metastasis & Lung $(61,62)$ \\
\hline circCEP128 & miR-145 & SOX11 & Cell proliferation & Bladder (63) \\
\hline circUBAP2 & miR-143 & Bcl-2 & Apoptosis & Osteosarcoma (64) \\
\hline circLAMP1 & miR-615 & DDR2 & Cell growth and apoptosis & T-cell lymphoma (24) \\
\hline circDLST & miR-502 & NRAS/MEK1/ERK1/2 & Cell invasion, DNA synthesis & Gastric (65) \\
\hline circBIRC6 & miR-3918 & $\mathrm{Bcl}-2$ & Apoptosis & $\mathrm{HCC}(25)$ \\
\hline
\end{tabular}

circRNA, circular RNA; miRNA/miR, microRNA; SRY, sex-determining region Y; PVT1, plasmacytoma variant translocation 1; CEP128, centrosomal protein $128 \mathrm{kDa}$; UBAP2, ubiquitin associated protein 2; LAMP1, lysosomal-associated membrane protein 1; DLST, dihydrolipoamide S-succinyltransferase; BIRC6, baculoviral IAP repeat containing 6; CRC, colorectal cancer; HCC, hepatocellular carcinoma.

Protein-circRNA interactions can also aid in suppressing tumourigenesis. Antisense non-coding RNA in the INK4 locus circRNA (circANRIL) binds to the c-terminal of pescadillo ribosomal biogenesis factor 1 (PES1) (72). PES1 is an RBP that mediates the processing of pre-mRNA through exonuclease activity and is essential for 60S ribosomal assembly. Through binding to PES1, circANRIL prevents its interactions with ribosomal RNA and impairs biogenesis of the ribosomes, leading in turn to an increase in p53 expression and apoptosis (70).

One circRNA that has been thoroughly studied in regards to protein binding is forkhead box O3 circRNA (circFOXO3), which binds to numerous proteins aiding in reducing cell growth and survival. In the cytoplasm, circFOXO3 binds to proteins including FAK, hypoxia-inducible factor $1 \alpha$, inhibitor of DNA binding 1 and E2F transcription factor 1, preventing their translocation to the nucleus and reducing their anti-cellular senescence functions (73). It can also bind to CDK2 and p21, forming a tertiary complex and acting as a sponge for CDK2, preventing its normal functions, which include enhancement of cyclin A expression and promotion of G1/S transition (74). Therefore, increased expression of circFOXO3 leads to decreased cell cycle progression.

circFOXO3 may also serve roles in cancer through its regulation of $\mathrm{FOXO} 3$, with circFOXO3 increasing protein levels of FOXO3. FOXO3 is considered to be a tumour suppressor, reducing cell motility and invasion; reduced levels of FOXO3 lead to increases in PTEN and increased AKT signalling, and FOXO3 is often reported as downregulated in breast cancer (75). circFOXO3 increases FOXO3 levels by binding to it and preventing its ubiquitination by mouse double minute 2 homolog (MDM2). This in turn leads to increases in its downstream target p53-upregulated modulator of apoptosis (PUMA), promoting cell apoptosis (76). Notably, circFOXO3 is also capable of acting as a scaffold for MDM2 and $\mathrm{p} 53$, promoting the ubiquitination-mediated degradation of p53 via MDM2, thereby repressing apoptosis. It is possible that by promoting MDM2 and p53 interactions, circFOXO3 reduces the interaction between MDM2 and FOXO3, leading to increases in PUMA (7). Overexpression of circFOXO3 leads to cell apoptosis in vitro and reduces tumour growth in vivo (76).

\section{Translated circRNAs}

Although defined as non-coding RNAs, there is some evidence suggesting that certain circRNAs may be translated. Originally, studies reported in principle and artificial systems, that circRNAs may be able to be translated using an internal ribosome entry site (IRES) (77,78). In subsequent years, experimental evidence has further supported this view. For example, it has been found that specific circRNAs that contain the same start codon as the gene they are derived from can associate with ribosomes (79). Secondly, a protein product derived from the Muscleblind (Mbl) transcript was also discovered that could only have originated from its circular form, translated using an IRES in the 3' UTR (77). Another circRNA proposed be translated is zinc finger protein 609 circRNA (circZNF609). Similar to the translated Mbl circRNA, circZNF609 contains the same start codon as its linear transcript and an in-frame stop codon; the transcript was reported to associate with ribosomes and be translated in a cap-independent manner using an IRES (79). Of note, Transcript Isoform in Polysomes sequencing showed $22 \%$ of all circRNAs investigated were associated with ribosomal fractions, suggesting that circRNAs may exhibit translatable activity (80). This polysome profiling also found that circRNAs in the ribosomal fractions mostly accumulated in the lighter fractions, suggesting circRNAs generally associate with fewer ribosomes along their length than linear mRNAs $(39,80)$. However, it must be noted that the vast majority of circRNAs appear to not be translated. These two translated circRNAs (Mb1 and circZNF609) are also found in brain and muscle cells, respectively, where circRNAs are expressed at higher levels; therefore, abundance of circRNA 
may contribute to transcript expression $(77,79)$. In addition, the RNA modification N6-methyladenosine $\left(\mathrm{m}^{6} \mathrm{~A}\right)$ was found to be enriched in these circRNAs (81). This modification aids in translation through recruitment of translation initiation factors, including eukaryotic translation initiation factor $4 \gamma 2$ and YTH domain-containing family protein 3 (82).

\section{Oncogenic viruses and circRNAs}

Viruses are estimated to be responsible for $20 \%$ of all global cancer cases, with 7 viruses currently classified as human oncogenic viruses: Hepatitis B virus (HBV); hepatitis C virus; Merkel cell polyomavirus; Epstein-Barr virus (EBV); Kaposi's sarcoma (KS)-associated herpesvirus (KSHV); human papilloma virus; and human T-lymphotrophic virus 1 (83). Of these viruses, circRNAs have been reported to be encoded by EBV and KSHV (84,85). In all viruses, only DNA viruses have been discovered to encode circRNAs, with members of the herpesviridae family thus far dominating. This is not unexpected, due to their larger genome size and complexity; herpesvirus genomes are known to undergo the complex splicing necessary for the biogenesis of circRNAs. Both EBV and KSHV also encode their own miRNAs; EBV encodes at least 44 miRNAs, whereas KSHV encodes $18(86,87)$. This suggests that both EBV and KSHV, by encoding their own miRNAs and circRNAs, seek to dysregulate the cellular non-coding RNA network.

EBV expresses circRNAs from its BART locus; these circRNAs include both Ecircs and EIcircs. These transcripts were found to be expressed in cells latently infected with EBV, including Burkitt's lymphoma and nasopharyngeal cancer cell lines (84), suggesting a potential role in EBV oncogenesis. Other circRNAs encoded by EBV may be expressed both in the latent and lytic lifecycles. For example, circBHLF1 was found to be expressed at low levels during latency with a large upregulation during reactivation, suggesting a potential role in regulating the lytic/latent switch, whereas circRPMS1_E4_ E3a and circRPMS1_E4_E2 were both found to be expressed in EBV-positive stomach cancer tissues (88).

$\mathrm{KSHV}$ has been found to constitutively express a circRNA from its vIRF4 locus, as well as hundreds of low-copy circRNAs from its PAN locus (84). The circRNAs originating from the PAN locus were highly variable, and the authors suggested that they may be merely artefacts from processing PAN RNA due to the high copy number.

Notably, when searching for circRNAs encoded by KSHV, the miRNAs these circRNA may sponge were found to be enriched in pathways involved with the regulation of apoptosis and cancer, suggesting the potential for regulating oncogenic processes (89). These circRNAs were found to be expressed during lytic replication and played roles in altering cell replication. Using seed sequence analysis, the KSHV circRNAs also exhibited the potential to sponge virally-encoded miRNAs in addition to cellular miRNAs. These circRNAs were found in KSHV-infected endothelial cells and from primary effusion lymphoma (PEL) cells. Furthermore, these circRNAs were also discovered in the lymph nodes of patients suffering from KSHV-associated malignancies, including KS, PEL and multicentric Castleman's disease (89). Finally, there is the potential for other herpesviruses to encode circRNAs, with the monkey homologue for EBV, rhesus macaque lymphocryptovirus, and another $\gamma$-herpesvirus, murine $\gamma$-herpesvirus 68 , both reported to encode circRNAs (85).

As well as encoding their own circRNAs, viruses have been shown to dysregulate cellular circRNAs to aid their survival and replication. The oncogenic virus, simian virus 40 (SV40), was reported to dysregulate 134 cellular circRNAs during infection, of which 103 were upregulated (90). These circRNAs were found to be involved in regulation pathways crucial to oncogenesis, including the p53 and Wnt/ $\beta$-catenin pathways. In addition, a potential circRNA:miRNA: mRNA regulatory network was identified, suggesting that SV40 is capable of dysregulating non-coding RNAs to aid viral manipulation of cellular gene expression, supporting viral replication (90).

As well as oncogenic DNA viruses dysregulating cellular circRNAs to aid in tumourigenesis, the retrovirus avian sarcoma leukosis virus (ASLV) has been found to dysregulate circVav3 (91). ASLV causes cancer in various types of bird, including chickens, pheasants and quails. circVav3 was found to be upregulated in liver tumours from ASLV-infected birds, where it acted as a sponge for gga-miR-375, leading to an increase in the expression of downstream mRNA targets. Among these targets is the transcription factor YAP1, which promotes cell proliferation and suppresses apoptosis. This sponging also led to increases in EMT activity and several EMT-associated markers, including N-cadherin and MMP2 (91). Furthermore, studies have shown differences in circRNA expression in ASLV-resistant and ASLV-susceptible birds, suggesting that circRNAs may either aid in suppressing oncogenesis or in tumour induction, depending on expression profiles (92).

Cellular circRNAs have also been reported as dysregulated by non-oncogenic viruses, suggesting that the mechanisms underlying virus-mediated dysregulation of circRNAs are varied and understudied, and that dysregulation of circRNAs is important in a range of diseases. For example, in early HIV infection, $>1,300$ circRNAs have been shown to be dysregulated, with 67 circRNAs proposed to serve a role in HIV replication (93). An RNA regulatory network of $>500$ dysregulated circRNAs, 900 dysregulated mRNAs and 21 miRNAs was also discovered. Similarly, a circRNA:miRNA network has also been identified during Ebola virus infection (94). Finally, herpes simplex virus 1 dysregulates $>500$ circRNAs and >207 miRNAs, once again emphasising the potential for dysregulation of the circRNA:miRNA axis to attenuate cellular gene transcription (95).

\section{0. circRNAs and exosomes}

Exosomes are nanosized extracellular vesicles that transport a wide variety of molecules, from nucleic acids and lipids to proteins and hormones, with crucial roles in cell communication. Exosomes have also been implicated in tumourigenesis through roles in mediating the tumour microenvironment, modulating the immune response and aiding metastasis (96). Mechanisms behind these roles including transporting extracellular matrix remodelling enzymes and growth promoting factors such as EGFR (97). Exosomes have also been shown to deliver miRNAs that promote oncogenesis, including miR-9 and miR-105 (98). 
Recent studies have provided evidence that exosomes may also transport and deliver circRNAs extracellularly; these so called exo-circRNAs may also have roles in both tumourigenesis and tumour suppression through delivery of circRNAs. CDR1as, the first functional circRNA discovered, has been shown to be delivered to distant cells in exosomes and once delivered to new cells, reduces the cellular levels of miR-7, inhibiting cell growth.

Furthermore, these exo-circRNAs may also act as functional biomarkers in a range of cancers. circRNAs were first shown to exist in exosomes in 2015, with $>1,000$ discovered in human serum exosomes alone, with the ratio of circRNAs to their respective linear transcripts found to be enriched in a number of exosomes (99). These exo-circRNA populations only mildly correlate with cellular populations, suggesting a separate mechanism for circRNA regulation or transportation into exosomes from the main cellular environment. Studies have shown that exo-circRNAs can be excreted by tumours into serum and fluid throughout the body; these exo-circRNAs have been isolated, and significant differences were found between their circRNA compositions compared with normal healthy cells $(100,101)$. Tumour exosomes from colorectal cancer had 257 novel circRNAs, and were missing 67 compared to exosomes from healthy tissues (99).

Specific exo-circRNA functions in cancer have also been investigated. Metastatic PDAC cells have been found to excrete a circRNA in exosomes, phosphodiesterase 8A circRNA (circPDE8A) (102). Increased levels of this circRNA are associated with poor prognosis, increased metastasis and increased lymphatic invasion. Once the exosome delivers circPDE8A to new cells, it can sponge miR-338. Downregulation of miR-338 leads to an increased in expression of metastasis-associated in colon cancer protein 1, in turn leading to activation of the MET/ERK and AKT signalling pathways, promoting cell growth and cell survival (102). This circRNA, once secreted into blood plasma inside of exosomes, was able to be detected, and may not only be a biomarker for PDAC cancer, but suggest the severity of the cancer. Specific exo-circRNAs have also been discovered to be associated with HCC, gastric and urothelial cancer (101).

\section{Biomarkers}

Although research into circRNAs remains in its infancy, these molecules possess the potential to become novel biomarkers for a range of diseases, from cancer to viral infections, due to their inherent properties. circRNAs are highly stable due to their resistance to exonucleases, and have a half-life of $>48 \mathrm{~h}$ compared with mRNA's half-life of $\sim 10 \mathrm{~h}$ (103). They are often disease-, tissue- and developmental stage-specific, allowing a precise diagnosis and even a prognosis. Furthermore, due to the identification of circRNAs in exosomes, this may enable the detection of disease-specific circRNAs in the blood, thereby allowing the detection of tumours early on through blood testing without the invasiveness associated with solid biopsies. As well as blood, circRNAs have been found enriched in a variety of body fluids including saliva, urine and cerebrospinal fluid (99). circRNAs have also been touted to be biomarkers in cardiovascular disease, neurological disease and diabetes (103). Infections also induce changes in cellular circRNAs, which could be used as a biomarkers; chronic HBV infection leads to the dysregulation of at least 99 circRNAs (104), whereas Mycobacterium Tuberculosis has been reported to dysregulate at least 61 cellular circRNAs during active infection (105). Of these, hsa_circ_0001953 and hsa_circ_0009024 have been suggested to be viable biomarkers, both displaying strong associations with active TB infection.

\section{Fusion circRNAs (f-circRNAs)}

The phenomenon of chromosomal translocation is a well-studied area in cancer biology, with numerous examples of translocations leading to the aberrant expression of oncogenic fusion proteins, most notably Bcr-Abl and promyelocytic leukaemia-retinoic acid receptor $\alpha$ (PML-RAR $\alpha$ ) (106). However, the effects chromosomal translocations have on the expression of circRNAs is yet to be determined. Recently, these f-circRNAs have been implicated in several cancers and oncogenic processes. These fusion circRNAs have two junctions, the junction where two genes far apart in the genome have joined together and the backsplice junction. It has been shown that in tumours caused by chromosomal translocations, 50\% expressed f-circRNAs, including PML-RAR $\alpha$ and nucleophosmin 1-anaplastic lymphoma kinase (NPM1-ALK) (107). NPM1-ALK-associated cancers have been shown to express f-circRNAs; these f-circRNAs are uniquely expressed only in the transformed cells and were not detected in wild-type cells (108). An f-circRNA, f-circAF9, was not only expressed in haematopoietic stem cells, but also led to a proliferative advantage and contributed to transformation of these cells, suggesting these f-circRNAs may serve active roles during oncogenic processes (109).

\section{Future prospects}

Long dismissed as simple by-products and errors in splicing, research into the roles and functions of circRNAs has shown that not only do they serve key roles in regulating gene expression in normal cells, but that their dysregulation can contribute to tumour development. Through their roles in miRNA sponging, protein sponging and gene expression, it is clear that circRNAs play an important role and further investigation is required. However, despite recent breakthroughs, a number of questions remain concerning circRNAs, with gaps in knowledge in all areas of circRNA research, particularly biogenesis, regulation and function. Regarding circRNA biogenesis, factors that aid circularisation, including longer flanking introns, nucleotide repeats and cellular RBPs have been identified; however, more unidentified factors undoubtedly remain and require further characterisation. Furthermore, the specific process of biogenesis remains poorly understood, with conflicting evidence suggesting circRNAs are synthesised both co- and post-transcriptionally. For example, circRNAs are often transcribed at a faster rate than linear RNAs, implying that regulation of RNA pol II and therefore its transcriptional speed may also play a role in their biogenesis (39). The export of Ecircs from the nucleus is also not fully elucidated. Initial research suggests specific cellular export factors, such as UAP56, regulate this process, but again which specific factors need to be identified. Finally, the stability of circRNAs is a 
crucial area that must be further investigated, as it contributes substantially to their function. Initial studies suggest that they are resistant to various RNases and capable of being exosomal markers (14). As such, several questions remain, namely how cells regulate and control their degradation. This is particularly relevant in respect to diseases such as cancer that can lead to the downregulation of specific circRNAs. As further research is conducted, the impact of circRNAs in cell regulation and in diseases, such as cancer, should be revealed. In particular, the potential for circRNAs to act as biomarkers or targets of novel therapeutic approaches merits further investigation.

\section{Acknowledgements}

The authors would like to thank all members of the Whitehouse laboratory for useful discussions.

\section{Funding}

This work was supported in part by grants from the White Rose BBSRC Doctoral Training Partnership in Mechanistic Biology (grant no. 95519935) and MRC DiMeN Doctoral Training Partnership (grant no. 95505183).

\section{Availability of data and materials}

Not applicable.

\section{Authors' contributions}

All authors contributed to designing and drafting this manuscript.

\section{Ethics approval and consent to participate}

Not applicable.

\section{Patient consent for publication}

Not applicable.

\section{Competing interests}

The authors declare that they have no competing interests.

\section{References}

1. Salzman J: Circular RNA expression: Its potential regulation and function. Trends Genet 32: 309-316, 2016.

2. Ebbesen KK, Hansen TB and Kjems J: Insights into circular RNA biology. RNA Biol 14: 1035-1045, 2017.

3. Taylor JM: Host RNA circles and the origin of hepatitis delta virus. World J Gastroenterol 20: 2971-2978, 2014.

4. Nigro JM, Cho KR, Fearon ER, Kern SE, Ruppert JM, Oliner JD, Kinzler KW and Vogelstein B: Scrambled exons. Cell 64: 607-613, 1991.

5. Jeck WR and Sharpless NE: Detecting and characterizing circular RNAs. Nat Biotechnol 32: 453-461, 2014.

6. Hansen TB, Jensen TI, Clausen BH, Bramsen JB, Finsen B, Damgaard CK and Kjems J: Natural RNA circles function as efficient microRNA sponges. Nature 495: 384-388, 2013.

7. Kristensen LS, Hansen TB, Ven $\varnothing$ MT and Kjems J: Circular RNAs in cancer: Opportunities and challenges in the field. Oncogene 37: 555-565, 2018
8. Chen LL and Yang L: Regulation of circRNA biogenesis. RNA Biol 12: 381-388, 2015.

9. Holdt LM, Kohlmaier A and Teupser D: Molecular roles and function of circular RNAs in eukaryotic cells. Cell Mol Life Sci 75: 1071-1098, 2018

10. Barrett SP and Salzman J: Circular RNAs: Analysis, expression and potential functions. Development 143: 1838-1847, 2016.

11. Liang D and Wilusz JE: Short intronic repeat sequences facilitate circular RNA production. Genes Dev 28: 2233-2247, 2014.

12. Xu S, Zhou L, Ponnusamy M, Zhang L, Dong Y, Zhang Y, Wang Q, Liu J and Wang K: A comprehensive review of circRNA: From purification and identification to disease marker potential. PeerJ 6: e5503, 2018.

13. Chen I, Chen CY and Chuang TJ: Biogenesis, identification, and function of exonic circular RNAs. Wiley Interdiscip Rev RNA 6: 563-579, 2015

14. Wang J, Zhu M, Pan J, Chen C, Xia S and Song Y: Circular RNAs: A rising star in respiratory diseases. Respir Res 20: 3, 2019.

15. Zhang Y, Zhang XO, Chen T, Xiang JF, Yin QF, Xing YH, Zhu S, Yang L and Chen LL: Circular intronic long noncoding RNAs. Mol Cell 51: 792-806, 2013

16. Huang S, Yang B, Chen BJ, Bliim N, Ueberham U, Arendt T and Janitz M: The emerging role of circular RNAs in transcriptome regulation. Genomics 109: 401-407, 2017.

17. Conn SJ, Pillman KA, Toubia J, Conn VM, Salmanidis M, Phillips CA, Roslan S, Schreiber AW, Gregory PA and Goodall GJ: The RNA binding protein quaking regulates formation of circRNAs. Cell 160: 1125-1134, 2015.

18. Huang C, Liang D, Tatomer DC and Wilusz JE: A length-dependent evolutionarily conserved pathway controls nuclear export of circular RNAs. Genes Dev 32: 639-644, 2018.

19. Schumann S, Jackson BR, Yule I, Whitehead SK, Revill C, Foster R and Whitehouse A: Targeting the ATP-dependent formation of herpesvirus ribonucleoprotein particle assembly as an antiviral approach. Nat Microbiol 2: 16201, 2016.

20. Ivanov A, Memczak S, Wyler E, Torti F, Porath HT, Orejuela MR, Piechotta M, Levanon EY, Landthaler M, Dieterich C and Rajewsky N: Analysis of intron sequences reveals hallmarks of circular RNA biogenesis in animals. Cell Rep 10: 170-177, 2015.

21. Aktaş T, AvşarIlık I, Maticzka D, Bhardwaj V,Pessoa Rodrigues C, Mittler G, Manke T, Backofen R and Akhtar A: DHX9 suppresses RNA processing defects originating from the Alu invasion of the human genome. Nature 544: 115-119, 2017

22. Chen D, Lu X, Yang F and Xing N: Circular RNA circHIPK3 promotes cell proliferation and invasion of prostate cancer by sponging miR-193a-3p and regulating MCL1 expression. Cancer Manag Res 11: 1415-1423, 2019.

23. Chen Y, Yang F, Fang E, Xiao W, Mei H, Li H, Li D, Song H, Wang J, Hong M, et al: Circular RNA circAGO2 drives cancer progression through facilitating HuR-repressed functions of AGO2-miRNA complexes. Cell Death Differ 26: 1346-1364, 2019.

24. Deng L, Liu G, Zheng C, Zhang L, Kang Y and Yang F: Circ-LAMP1 promotes T-cell lymphoblastic lymphoma progression via acting as a ceRNA for miR-615-5p to regulate DDR 2 expression. Gene 701: 146-151, 2019.

25. Yang G, Wang X, Liu B, Lu Z, Xu Z, Xiu P, Liu Z and Li J: circ-BIRC6, a circular RNA, promotes hepatocellular carcinoma progression by targeting the miR-3918/Bcl2 axis. Cell Cycle 18: 976-989, 2019.

26. Wu Q, Li P, Wu M and Liu Q: Deregulation of circular RNAs in cancer from the perspectives of aberrant biogenesis, transport and removal. Front Genet 10: 16, 2019.

27. Wang C, Zou J, Ma X, Wang E and Peng G: Mechanisms and implications of ADAR-mediated RNA editing in cancer. Cancer Lett 411: 27-34, 2017.

28. Lee T and Pelletier J: The biology of DHX9 and its potential as a therapeutic target. Oncotarget 7: 42716-42739, 2016.

29. Goehe RW, Shultz JC, Murudkar C, Usanovic S, Lamour NF, Massey DH, Zhang L, Camidge DR, Shay JW, Minna JD and Chalfant CE: hnRNP L regulates the tumorigenic capacity of lung cancer xenografts in mice via caspase-9 pre-mRNA processing. J Clin Invest 120: 3923-3939, 2010.

30. Gou Q, Wu K, Zhou JK, Xie Y, Liu L and Peng Y: Profiling and bioinformatic analysis of circular RNA expression regulated by c-Myc. Oncotarget 8: 71587-71596, 2017.

31. Panda AC: Circular RNAs act as miRNA sponges. Adv Exp Med Biol 1087: 67-79, 2018

32. O'Brien J, Hayder H, Zayed Y and Peng C: Overview of MicroRNA biogenesis, mechanisms of actions, and circulation. Front Endocrinol (Lausanne) 9: 402, 2018. 
33. Rong D, Sun H, Li Z, Liu S, Dong C, Fu K, Tang W and Cao H: An emerging function of circRNA-miRNAs-mRNA axis in human diseases. Oncotarget 8: 73271-73281, 2017.

34. Mitra A, Pfeifer K and Park KS: Circular RNAs and competing endogenous RNA (ceRNA) networks. Transl Cancer Res 7 (Suppl 5): S624-S628, 2018.

35. Liu L, Wang J, Khanabdali R, Kalionis B, Tai X and Xia S: Circular RNAs: Isolation, characterization and their potential role in diseases. RNA Biol 14: 1715-1721, 2017.

36. Leggio L, Vivarelli S, L'Episcopo F, Tirolo C, Caniglia S, Testa N, Marchetti B and Iraci N: microRNAs in Parkinson's disease: From pathogenesis to novel diagnostic and therapeutic approaches. Int J Mol Sci 18: E2698, 2017.

37. Rothman A, Restrepo H, Sarukhanov V, Evans WN, Wiencek RG Jr, Williams R, Hamburger N, Anderson K, Balsara J and Mann D: Assessment of microRNA and gene dysregulation in pulmonary hypertension by endoarterial biopsy. Pulm Circ 7: 455-464, 2017.

38. Girardi E, López P and Pfeffer S: On the importance of Host MicroRNAs during viral infection. Front Genet 9: 439, 2018

39. Ragan C, Goodall GJ, Shirokikh NE and Preiss T: Insights into the biogenesis and potential functions of exonic circular RNA. Sci Rep 9: 2048, 2019.

40. Wilusz JE: A $360^{\circ}$ view of circular RNAs: From biogenesis to functions. Wiley Interdiscip Rev RNA 9: e1478, 2018.

41. Quan G and Li J: Circular RNAs: Biogenesis, expression and their potential roles in reproduction. J Ovarian Res 11: 9, 2018.

42. Kulcheski FR, Christoff AP and Margis R: Circular RNAs are miRNA sponges and can be used as a new class of biomarker J Biotechnol 238: 42-51, 2016.

43. Pratt AJ and MacRae IJ: The RNA-induced silencing complex: A versatile gene-silencing machine. J Biol Chem 284: 17897-17901, 2009.

44. Kluiver J, Gibcus JH, Hettinga C, Adema A, Richter MK, Halsema N, Slezak-Prochazka I, Ding Y, Kroesen BJ and van den Berg A: Rapid generation of microRNA sponges for microRNA inhibition. PLoS One 7: e29275, 2012.

45. Memczak S, Jens M, Elefsinioti A, Torti F, Krueger J, Rybak A, Maier L, Mackowiak SD, Gregersen LH, Munschauer M, et al: Circular RNAs are a large class of animal RNAs with regulatory potency. Nature 495: 333-338, 2013.

46. Peng L, Yuan XQ and Li GC: The emerging landscape of circular RNA ciRS-7 in cancer (Review). Oncol Rep 33: 2669-2674, 2015.

47. Liu L, Liu FB, Huang M, Xie K, Xie QS, Liu CH, Shen MJ and Huang Q: Circular RNA ciRS-7 promotes the proliferation and metastasis of pancreatic cancer by regulating miR-7-mediated EGFR/STAT3 signaling pathway. Hepatobiliary Pancreat Dis Int: Mar 9: S1499-3872(19)30039-6, 2019 (Epub ahead of print) doi: 10.1016/j.hbpd.2019.03.003.

48. Zheng Q, Bao C, Guo W, Li S, Chen J, Chen B, Luo Y, Lyu D, Li Y, Shi G, et al: Circular RNA profiling reveals an abundant circHIPK 3 that regulates cell growth by sponging multiple miRNAs. Nat Commun 7: 11215, 2016.

49. Zeng K, Chen X, Xu M, Liu X, Hu X, Xu T, Sun H, Pan Y, He B and Wang S: CircHIPK3 promotes colorectal cancer growth and metastasis by sponging miR-7. Cell Death Dis 9: 417, 2018.

50. Chen G, Shi Y, Liu M and Sun J: circHIPK3 regulates cell proliferation and migration by sponging miR-124 and regulating AQP3 expression in hepatocellular carcinoma. Cell Death Dis 9: $175,2018$.

51. $\mathrm{Yu} \mathrm{H}$, Chen $\mathrm{Y}$ and Jiang P: Circular RNA HIPK3 exerts oncogenic properties through suppression of miR-124 in lung cancer. Biochem Biophys Res Commun 506: 455-462, 2018.

52. Morgan EL, Wasson CW, Hanson L, Kealy D, Pentland I, McGuire V, Scarpini C, Coleman N, Arthur JSC, Parish JL, et al: STAT3 activation by E6 is essential for the differentiation-dependent HPV18 life cycle. PLoS Pathog 14 e1006975, 2018

53. Cheng J, Zhuo H, Xu M, Wang L, Xu H, Peng J, Hou J, Lin L and Cai J: Regulatory network of circRNA-miRNA-mRNA contributes to the histological classification and disease progression in gastric cancer. J Transl Med 16: 216, 2018.

54. Zhang J, Liu H, Hou L, Wang G, Zhang R, Huang Y, Chen X and Zhu J: Circular RNA_LARP4 inhibits cell proliferation and invasion of gastric cancer by sponging miR-424-5p and regulating LATS1 expression. Mol Cancer 16: 151, 2017.

55. Chen Z, Zuo X, Pu L, Zhang Y, Han G, Zhang L, Wu J and Wang X: circLARP4 induces cellular senescence through regulating miR-761/RUNX3/p53/p21 signaling in hepatocellular carcinoma. Cancer Sci 110: 568-581, 2019.
56. Levanon D, Bernstein Y, Negreanu V, Bone KR, Pozner A, Eilam R, Lotem J, Brenner O and Groner Y: Absence of Runx3 expression in normal gastrointestinal epithelium calls into question its tumour suppressor function. EMBO Mol Med 3: 593-604, 2011.

57. Wan L, Zhang L, Fan K, Cheng ZX, Sun QC and Wang JJ: Circular RNA-ITCH suppresses lung cancer proliferation via inhibiting the Wnt $/ \beta$-catenin pathway. Biomed Res Int 2016: 1579490, 2016

58. Yang C, Yuan W, Yang X, Li P, Wang J, Han J, Tao J, Li P, Yang H, Lv Q and Zhang W: Circular RNA circ-ITCH inhibits bladder cancer progression by sponging $\mathrm{miR}-17 / \mathrm{miR}-224$ and regulating p21, PTEN expression. Mol Cancer 17: 19, 2018

59. Huang X, Li Z, Zhang Q, Wang W, Li B, Wang L, Xu Z, Zeng A, Zhang X, Zhang X, et al: Circular RNA AKT3 upregulates PIK3R1 to enhance cisplatin resistance in gastric cancer via miR-198 suppression. Mol Cancer 18: 71, 2019.

60. Zhao ZJ and Shen J: Circular RNA participates in the carcinogenesis and the malignant behavior of cancer. RNA Biol 14: 514-521, 2017

61. Panda AC, Grammatikakis I, Kim KM, De S, Martindale JL, Munk R, Yang X, Abdelmohsen K and Gorospe M: Identification of senescence-associated circular RNAs (SAC-RNAs) reveals senescence suppressor CircPVT1. Nucleic Acids Res 45: 4021-4035, 2017

62. Li X, Zhang Z, Jiang H, Li Q, Wang R, Pan H, Niu Y, Liu F, Gu H, Fan X and Gao J: Circular RNA circPVT1 promotes proliferation and invasion through sponging miR-125b and activating E2F2 signaling in non-small cell lung cancer. Cell Physiol Biochem 51: 2324-2340, 2018

63. Wu Z, Huang W, Wang X, Wang T, Chen Y, Chen B, Liu R, Bai P and Xing J: Circular RNA CEP128 acts as a sponge of miR-145-5p in promoting the bladder cancer progression via regulating SOX11. Mol Med 24: 40, 2018.

64. Zhang H, Wang G, Ding C, Liu P, Wang R, Ding W, Tong D, Wu D, Li C, Wei Q, et al: Increased circular RNA UBAP2 acts as a sponge of miR-143 to promote osteosarcoma progression. Oncotarget 8: 61687-61697, 2017.

65. Zhang J, Hou L, Liang R, Chen X, Zhang R, Chen W and Zhu J: CircDLST promotes the tumorigenesis and metastasis of gastric cancer by sponging miR-502-5p and activating the NRAS/ MEK1/ERK1/2 signaling. Mol Cancer 18: 80, 2019.

66. Li Z, Huang C, Bao C, Chen L, Lin M, Wang X, Zhong G, Yu B, $\mathrm{Hu}$ W, Dai L, et al: Exon-intron circular RNAs regulate transcription in the nucleus. Nat Struct Mol Biol 22: 256-264, 2015

67. Holdt LM, Kohlmaier A and Teupser D: Molecular functions and specific roles of circRNAs in the cardiovascular system. Noncoding RNA Res 3: 75-98, 2018.

68. Qin M, Wei G and Sun X: Circ-UBR5: An exonic circular RNA and novel small nuclear RNA involved in RNA splicing. Biochem Biophys Res Commun 503: 1027-1034, 2018.

69. Abdelmohsen K, Panda AC, Munk R, Grammatikakis I, Dudekula DB, De S, Kim J, Noh JH, Kim KM, Martindale JL and Gorospe M: Identification of HuR target circular RNAs uncovers suppression of PABPN1 translation by CircPABPN1. RNA Biol 14: 361-369, 2017.

70. Holdt LM, Stahringer A, Sass K, Pichler G, Kulak NA, Wilfert W, Kohlmaier A, Herbst A, Northoff BH, Nicolaou A, et al: Circular non-coding RNA ANRIL modulates ribosomal RNA maturation and atherosclerosis in humans. Nat Commun 7: 12429, 2016.

71. Banerjee A, Apponi LH, Pavlath GK and Corbett AH: PABPN1: Molecular function and muscle disease. FEBS J 280: 4230-4250, 2013.

72. Lapik YR, Fernandes CJ, Lau LF and Pestov DG: Physical and functional interaction between Pes1 and Bop1 in mammalian ribosome biogenesis. Mol Cell 15: 17-29, 2004.

73. Du WW, Yang W, Chen Y, Wu ZK, Foster FS, Yang Z, Li X and Yang BB: Foxo3 circular RNA promotes cardiac senescence by modulating multiple factors associated with stress and senescence responses. Eur Heart J 38: 1402-1412, 2017.

74. Du WW, Yang W, Liu E, Yang Z, Dhaliwal P and Yang BB: Foxo3 circular RNA retards cell cycle progression via forming ternary complexes with p21 and CDK2. Nucleic Acids Res 44: 2846-2858, 2016

75. Lu WY: Roles of the circular RNA circ-Foxo3 in breast cancer progression. Cell Cycle 16: 589-590, 2017.

76. Du WW, Fang L, Yang W, Wu N, Awan FM, Yang Z and Yang BB: Induction of tumor apoptosis through a circular RNA enhancing Foxo3 activity. Cell Death Differ 24: 357-370, 2017. 
77. Pamudurti NR, Bartok O, Jens M, Ashwal-Fluss R, Stottmeister C Ruhe L, Hanan M, Wyler E, Perez-Hernandez D, Ramberger E, et al: Translation of CircRNAs. Mol Cell 66: 9-21.e7, 2017.

78. Wesselhoeft RA, Kowalski PS and Anderson DG: Engineering circular RNA for potent and stable translation in eukaryotic cells. Nat Commun 9: 2629, 2018.

79. Legnini I, Di Timoteo G, Rossi F, Morlando M, Briganti F, Sthandier O, Fatica A, Santini T, Andronache A, Wade M, et al: Circ-ZNF609 is a circular RNA that can be translated and functions in myogenesis. Mol Cell 66: 22-37.e9, 2017.

80. Floor SN and Doudna JA: Tunable protein synthesis by transcript isoforms in human cells. Elife 5: e10921, 2016.

81. Zhou C, Molinie B, Daneshvar K, Pondick JV, Wang J, Van Wittenberghe N, Xing Y, Giallourakis CC and Mullen AC: Genome-wide maps of m6A circRNAs identify widespread and cell-type-specific methylation patterns that are distinct from mRNAs. Cell Rep 20: 2262-2276, 2017.

82. Arnaiz E, Sole C, Manterola L, Iparraguirre L, Otaegui D and Lawrie CH: CircRNAs and cancer: Biomarkers and master regulators. Semin Cancer Biol: S1044-579X(18)30099-3, 2018 Doi: 10.1016/j.semcancer.2018.12.002.

83. Luo GG and Ou JH: Oncogenic viruses and cancer. Virol Sin 30 $83-84,2015$

84. Toptan T, Abere B, Nalesnik MA, Swerdlow SH, Ranganathan S, Lee N, Shair KH, Moore PS and Chang Y: Circular DNA tumor viruses make circular RNAs. Proc Natl Acad Sci USA 115 E8737-E8745, 2018.

85. Ungerleider NA, Jain V, Wang Y, Maness NJ, Blair RV, Alvarez X, Midkiff C, Kolson D, Bai S, Roberts C, et al: Comparative analysis of gammaherpesvirus circular RNA repertoires: Conserved and unique viral circular RNAs. J Virol 93: e01952-18, 2019.

86. Wang M, Yu F, Wu W, Wang Y, Ding H and Qian L: Epstein-Barr virus-encoded microRNAs as regulators in host immune responses. Int J Biol Sci 14: 565-576, 2018.

87. Qin Z, Jakymiw A, Findlay V and Parsons C: KSHV-encoded MicroRNAs: Lessons for viral cancer pathogenesis and emerging concepts. Int J Cell Biol 2012: 603961, 2012.

88. Ungerleider N, Concha M, Lin Z, Roberts C, Wang X, Cao S, Baddoo M, Moss WN, Yu Y, Seddon M, et al: The Epstein Barr virus circRNAome. PLoS Pathog 14: e1007206, 2018.

89. Tagawa T, Gao S, Koparde VN, Gonzalez M, Spouge JL, Serquiña AP, Lurain K, Ramaswami R, Uldrick TS, Yarchoan R and Ziegelbauer JM: Discovery of Kaposi's sarcoma herpesvirus-encoded circular RNAs and a human antiviral circular RNA. Proc Natl Acad Sci USA 115: 12805-12810, 2018

90. Shi J, Hu N, Li J, Zeng Z, Mo L, Sun J, Wu M and Hu Y: Unique expression signatures of circular RNAs in response to DNA tumor virus SV40 infection. Oncotarget 8: 98609-98622, 2017.

91. Zhang X, Yan Y, Lin W, Li A, Zhang H, Lei X, Dai Z, Li X, Li H, Chen W, et al: Circular RNA Vav3 sponges gga-miR-375 to promote epithelial-mesenchymal transition. RNA Biol 16: 118-132, 2019.

92. Zhang X, Yan Y, Lei X, Li A, Zhang H, Dai Z, Li X, Chen W, Lin W, Chen F, et al: Circular RNA alterations are involved in resistance to avian leukosis virus subgroup-J-induced tumor formation in chickens. Oncotarget 8: 34961-34970, 2017.

93. Zhang Y, Zhang H, An M, Zhao B, Ding H, Zhang Z, He Y, Shang $\mathrm{H}$ and Han $\mathrm{X}$ : Crosstalk in competing endogenous RNA networks reveals new circular RNAs involved in the pathogenesis of early HIV infection. J Transl Med 16: 332, 2018.
94. Wang ZY, Guo ZD, Li JM, Zhao ZZ, Fu YY, Zhang CM, Zhang Y, Liu LN, Qian J and Liu LN: Genome-wide search for competing endogenous RNAs responsible for the effects induced by Ebola virus replication and transcription using a trVLP system. Front Cell Infect Microbiol 7: 479, 2017.

95. Shi J, Hu N, Mo L, Zeng Z, Sun J and Hu Y: Deep RNA sequencing reveals a repertoire of human fibroblast circular RNAs associated with cellular responses to Herpes simplex virus 1 infection. Cell Physiol Biochem 47: 2031-2045, 2018.

96. Tung KH, Ernstoff MS, Allen C and Shu S: A review of exosomes and their role in the tumor microenvironment and host-tumor 'Macroenvironment'. J Immunol Sci 3: 4-8, 2019.

97. Zheng H, Zhan Y, Liu S, Lu J, Luo J, Feng J and Fan S: The roles of tumor-derived exosomes in non-small cell lung cancer and their clinical implications. J Exp Clin Cancer Res 37: 226, 2018

98. Tai YL, Chen KC, Hsieh JT and Shen TL: Exosomes in cancer development and clinical applications. Cancer Sci 109: 2364-2374, 2018.

99. Li Y, Zheng Q, Bao C, Li S, Guo W, Zhao J, Chen D, Gu J, He X and Huang S: Circular RNA is enriched and stable in exosomes: A promising biomarker for cancer diagnosis. Cell Res 25: 981-984, 2015

100. Wang J, Zhang Q, Zhou S, Xu H, Wang D, Feng J, Zhao J and Zhong S: Circular RNA expression in exosomes derived from breast cancer cells and patients. Epigenomics 11: 411-421, 2019.

101. Bai H, Lei K, Huang F, Jiang Z and Zhou X: Exo-circRNAs: A new paradigm for anticancer therapy. Mol Cancer 18: 56, 2019.

102. Li Z, Yanfang W, Li J, Jiang P, Peng T, Chen K, Zhao X, Zhang Y, Zhen P, Zhu J and Li X: Tumor-released exosomal circular RNA PDE8A promotes invasive growth via the miR-338/MACC1/ MET pathway in pancreatic cancer. Cancer Lett 432: 237-250, 2018.

103.Zhang Z, Yang T and Xiao J: Circular RNAs: Promising biomarkers for human diseases. EBioMedicine 34: 267-274, 2018.

104. Wang M, Yu F and Li P: Circular RNAs: Characteristics, function and clinical significance in hepatocellular carcinoma. Cancers (Basel) 10: E258, 2018.

105. Huang Z, Su R, Qing C, Peng Y, Luo Q and Li J: Plasma circular RNAs hsa_circ_0001953 and hsa_circ_0009024 as diagnostic biomarkers for active tuberculosis. Front Microbiol 9: 2010, 2018.

106. De Braekeleer E, Douet-Guilbert $\mathrm{N}$ and De Braekeleer M: RARA fusion genes in acute promyelocytic leukemia: A review. Expert Rev Hematol 7: 347-357, 2014

107. Dal Molin A, Bresolin S, Gaffo E, Tretti C, Boldrin E, Meyer LH, Guglielmelli P, Vannucchi AM, Te Kronnie G and Bortoluzzi S: CircRNAs are here to stay: A perspective on the MLL recombinome. Front Genet 10: 88, 2019.

108. Babin L, Piganeau M, Renouf B, Lamribet K, Thirant C, Deriano L, Mercher T, Giovannangeli $\mathrm{C}$ and Brunet EC: Chromosomal translocation formation is sufficient to produce fusion circular RNAs specific to patient tumor cells. iScience 5: $19-29,2018$

109.Guarnerio J, Bezzi M, Jeong JC, Paffenholz SV, Berry K, Naldini MM, Lo-Coco F, Tay Y, Beck AH and Pandolfi PP: Oncogenic role of Fusion-circRNAs derived from cancer-associated chromosomal translocations. Cell 166: 1055-1056, 2016. 